\title{
Cardiopulmonary Affection in Patients with Systemic Lupus Erythematosus
}

Mohammed Mahmoud Nussier ${ }^{1}$, Mohamed Elwan Sayed ${ }^{2}$, Hosni Abd-Elkareem Younus ${ }^{1}$

Departments of ${ }^{1}$ Internal Medicine, ${ }^{2}$ Rheumatology and Rehabilitation, Assuit Faculty of Medicine, Al-Azhar

University, Egypt

*Correspondence author: Mohammed Mahmoud Nussier, Mobile: (+20) 01016873338

\begin{abstract}
Background; Systemic lupus erythematosus (SLE) is a systemic autoimmune disease that can affect any part of the body. Early detection and quantification of pathological changes are important for assessing the benefits of cardiopulmonary prevention in SLE management.

Objective: The aim was to study the effect of SLE on cardiopulmonary system, and its early detection.

Patients and Methods: Fifty patients were selected from those attending the outpatient clinics and inpatients who were admitted to Internal Medicine Department of Al-Azhar Assuit University Hospital, from May 2017 to May 2018, and fulfilled the American College of Rheumatology (ACR) 1982 revised criteria for classification of SLE. All patients were subjected to complete history taking, clinical examination, routine investigations, transthoracic echocardiography (Echo) and computed tomography (CT) of the chest.

Results: The most common echo finding was pericardial effusion seen in 20 patients (40\%), followed by mitral regurgitation in 14 patients (28\%), mitral valve prolapse was seen in 13 patients (26\%). The most common CT chest findings was ground glass opacity seen in 15 patients (30\%) followed by pleural effusion seen in 14 patients (28\%) and pleural thickening in 10 patients (20\%). There was non- significant correlation between EF\% and SLE Disease Activity Index (SLEDAI), but a negative significant correlation between disease duration and $\mathrm{EF} \%(p=0.02)$.

Conclusion: All SLE patients even who have clinically inactive disease should be screened for the presence of structural cardiac and chest abnormalities. Echocardiography and CT chest can be helpful as a noninvasive diagnostic tool for early detection of such abnormalities
\end{abstract}

Keywords: Cardiac changes, pulmonary changes, Systemic Lupus Erythematosus.

\section{INTRODUCTION}

SLE is a systemic autoimmune disease that can affect any part of the body. As occurs in other autoimmune diseases, the immune system attacks the body`s cells and tissue, resulting in inflammation and tissue damage ${ }^{(1)}$. The course of the disease is unpredictable, with periods of activity alternating with remissions. The disease occurs nine times more often in women that in men, especially in women in child-bearing years ages 15 to $35^{(2)}$. Patients with SLE have a high risk of cardiovascular disease. Coronary artery disease, myocarditis, endocarditis, valvular disease and pericarditis are major manifestations of cardiac involvement in SLE patients. Multiple SLEspecific mechanisms, such as autoimmune responses, altered autoantibody and cytokine levels, and lipid dysfunctions can accelerate the progression of overall atherosclerotic burden. Therefore, early detection and quantification of pathological changes are important for assessing the benefits of cardiovascular prevention in SLE management ${ }^{(3)}$. The respiratory system is more commonly affected in SLE than in any other systemic autoimmune disease and that all its components may be affected. Pleuropulmonary involvement was defined as the presence of one or more of the following manifestations: pleuritis, pneumonitis, pulmonary arterial hypertension, shrinking lung syndrome, pulmonary fibrosis, pulmonary hemorrhage, pulmonary thrombosis and lung infarction ${ }^{(4)}$. Disease activity varies over time and, at onset, symptoms are nonspecific and may include unexplained fever, extreme fatigue, muscle and joint pain and skin rash. SLE can presented by arthritis, nephritis, heart and lung inflammation, central nervous abnormalities and blood disorders ${ }^{(5)}$.

The aim was to study the effect of SLE on cardiopulmonary system, and its early detection.

\section{PATIENTS AND METHODS}

This study was carried out on 50 patients (44 females and 6 males), aged 16-56 years, and the mean age was $31.12 \pm 10.32$ years. The mean disease duration was (11.50 \pm 7.054$)$. The study was approved by local Ethical Committee of Assuit Faculty of medicine, AlAzhar University.

All patients fulfilled the American college of rheumatology (ACR) 1982 revised criteria for classification of SLE. These patients were selected from those attending outpatient clinics and inpatients who were admitted to the Internal Medicine Department of AlAzhar Assuit University Hospital, from May 2017 to May 2018. Informed and written consent was taken from all patients.

Exclusion criteria: Diabetic patients, patients with associated rheumatic diseases and pregnant women.

\section{All patients were subjected to the following: \\ A- History taking and Clinical examination:}

Socio-demographic data including age, sex, occupation, residence, marital status and special habits. History of deep venous thrombosis. $\square$ If married female: history of recurrent abortion. $\square$ History of fever, skin rash, purpuric eruption, ecchymotic patches, oral ulceration, photosensitivity, hair loss, arthralgia or arthritis and myalgia. 
Full physical examination.

B-Laboratory investigations: Complete blood picture, antinuclear antibody (ANA), anti double stranded DNA, CRP, ESR.

C-Transthoracic echocardiographic examination at rest: To assess the following parameters: left ventricular ejection fraction (EF), diastolic function, valvular state, pericardial state and pulmonary hypertension.

D- Computed tomography (CT) of the chest to assess the following: Pulmonary parenchymal changes, pulmonary interstitial changes, pulmonary vascular changes and mediastinal and pleural changes

E- Measurement of activity index: The damage was measured using the SLICC (Systemic Lupus International Collaborating Clinics)/ACR damage index (SDI). The disease flare was defined by the increase in the SLE Disease Activity Index ${ }^{(6)}$.

\section{Statistical analysis:}

Recorded data were analyzed using the statistical package for social sciences, version 20.0 (SPSS Inc., Chicago, Illinois, USA). Quantitative data were expressed as mean \pm standard deviation (SD). Qualitative data were expressed as frequency and percentage.

- Correlation coefficient was calculated. P-value <0.05 was considered significant.

\section{RESULTS}

This study was carried out on 50 patients ( 44 females and 6 males), aged $16-56$ years, and the mean age was $31.12 \pm$ 10.32 years (Table 1). Of the 50 patients, 45 were positive for antinuclear antibody and 30 patients were positive for anti DNA antibody.

Table (1) Distribution of study patients as regard clinical parameters

\begin{tabular}{|c|c|c|c|c|}
\hline Parameter & Minimum & Maximum & Mean & $\begin{array}{c}\text { Std. } \\
\text { Deviation }\end{array}$ \\
\hline Age (years) & 16 & 56 & 31.12 & 10.323 \\
\hline $\begin{array}{c}\text { Disease duration } \\
\text { (years) }\end{array}$ & 2 & 25 & 11.50 & 7.054 \\
\hline SBP (mmHg) & 100 & 180 & 138.60 & 21.854 \\
\hline DBP (mmHg) & 60 & 110 & 83.70 & 13.990 \\
\hline HR (beats/min.) & 66 & 120 & 89.80 & 15.413 \\
\hline SLEDAI Score & 6 & 22 & 12.88 & 4.601 \\
\hline
\end{tabular}

Echocardiography was done in all patients (Tables 2 and 3).

Table (2) Echocardiographic findings among study patients

\begin{tabular}{|c|c|c|}
\hline chocardiographic findings & Frequency & Percent \\
\hline LVH & 10 & $20 \%$ \\
\hline Diastolic dysfunction & 5 & $10 \%$ \\
\hline Regional hypokinesia & 6 & $12 \%$ \\
\hline Pulmonary HTN & 4 & $8 \%$ \\
\hline TR & 4 & $8 \%$ \\
\hline AR & 3 & $6 \%$ \\
\hline MVP & 13 & $26 \%$ \\
\hline MR & 14 & $28 \%$ \\
\hline
\end{tabular}

Table (3) Pericardial effusion among study patients

\begin{tabular}{|c|c|c|}
\hline \multicolumn{3}{|c|}{ Pericardial effusion } \\
\hline & Frequency & Percent \\
\hline No & 30 & $60 \%$ \\
\hline Mild & 7 & $14 \%$ \\
\hline Moderate & 8 & $16 \%$ \\
\hline Severe & 5 & $10 \%$ \\
\hline
\end{tabular}

As regard CT chest, there was 13 patients (26\%) had normal CT chest. The most common CT chest findings was ground glass opacity seen in 15 patients (Table 4).

Table (4) CT findings among study patients

\begin{tabular}{|c|c|c|}
\hline C T Findings & Frequency & Percent \\
\hline $\begin{array}{c}\text { Mediastinal lymph node } \\
\text { enlargement }\end{array}$ & $\mathbf{5}$ & $\mathbf{1 0 \%}$ \\
\hline Pleural effusion & $\mathbf{1 4}$ & $\mathbf{2 8 \%}$ \\
\hline Pleural thickening & $\mathbf{1 0}$ & $\mathbf{2 0 \%}$ \\
\hline $\begin{array}{c}\text { Pulmonary artery trunk } \\
\text { broadening }\end{array}$ & $\mathbf{5}$ & $\mathbf{1 0 \%}$ \\
\hline Bronchiectasis & $\mathbf{5}$ & $\mathbf{1 0 \%}$ \\
\hline Emphysema & $\mathbf{1}$ & $\mathbf{2 \%}$ \\
\hline $\begin{array}{c}\text { Interlobular septal } \\
\text { thickening }\end{array}$ & $\mathbf{9}$ & $\mathbf{1 8 \%}$ \\
\hline Honeycomb opacity & $\mathbf{4}$ & $\mathbf{8 \%}$ \\
\hline Ground glass opacity & $\mathbf{1 5}$ & $\mathbf{3 0 \%}$ \\
\hline Consolidation & $\mathbf{5}$ & $\mathbf{1 0 \%}$ \\
\hline
\end{tabular}

There was a significant correlation between disease duration and $\mathrm{EF} \%$ ( $\mathrm{p}=0.02$, figure 1). But There was nonsignificant correlation between disease duration and SLEDAI $(p=0.55$, figure 2$)$. Also there was nonsignificant correlation between EF\% and SLEDAI ( $\mathrm{p}=$ 0.95 , figure 3 ).

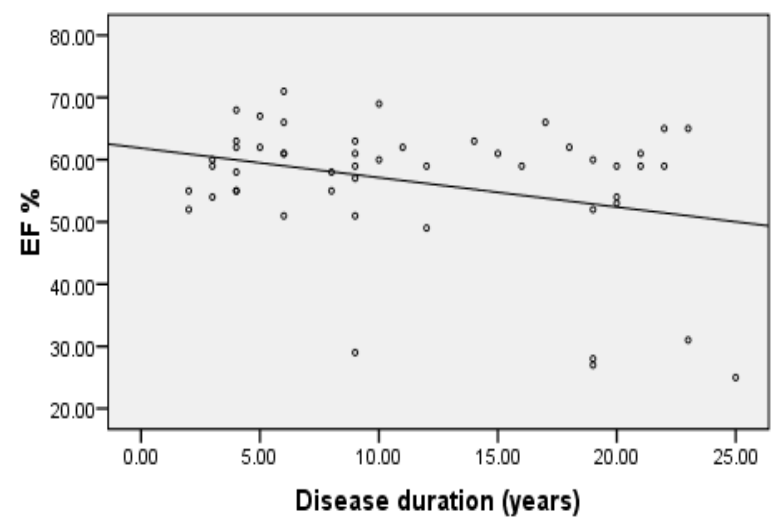

$R$ Sq Linear $=0$

Figure (1) Correlation between disease duration and EF\%.

There was a significant correlation

$$
(\mathrm{r}=-0.31 \mathrm{p}=0.02)
$$




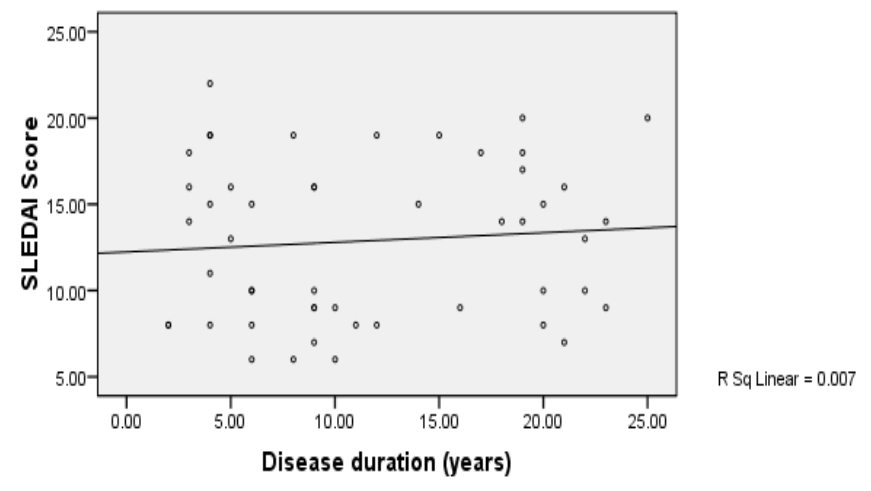

Figure (2) Correlation between disease duration and SLEDAI.

\section{There was non-significant correlation}

$$
(r=0.08 \mathrm{p}=0.55)
$$

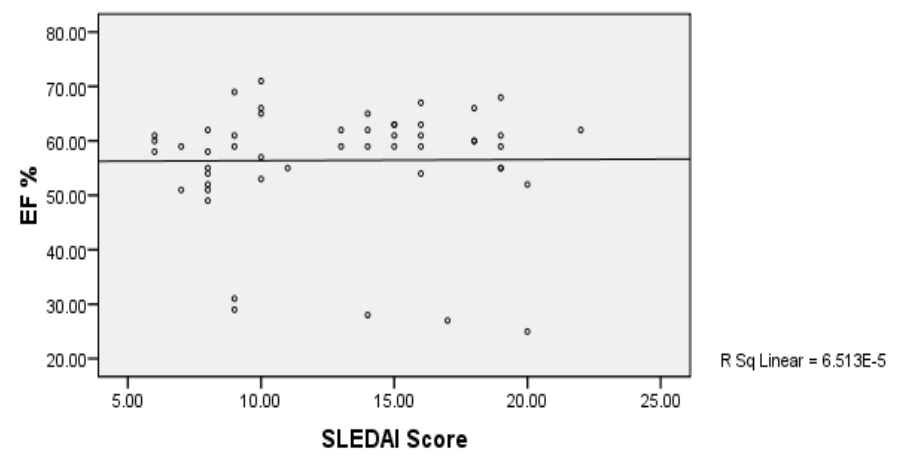

Figure (3) Correlation between EF\% and SLEDAI.

There was non-significant correlation

$$
(\mathrm{r}=0.008 \mathrm{p}=0.95)
$$

There was a significant correlation between CRP and SLEDAI ( $p=0.01$, figure 4$)$. But There was nonsignificant correlation between ESR and SLEDAI ( $\mathrm{p}=$ 0.92 , figure 5 ).

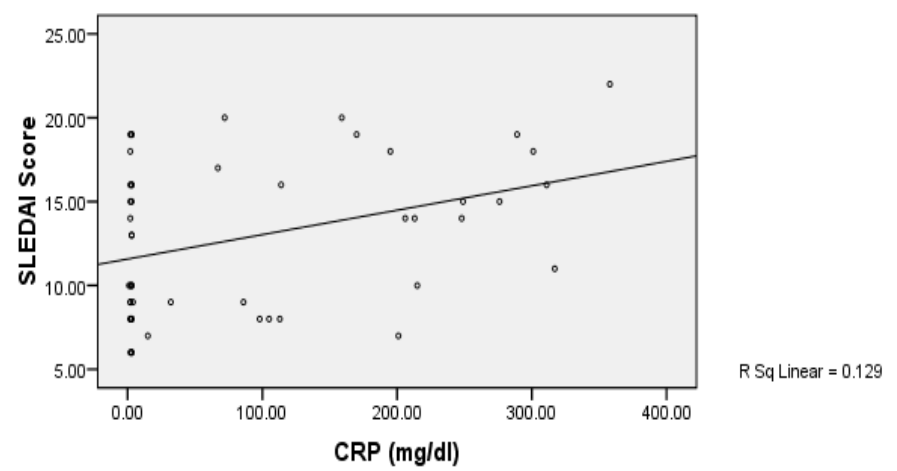

Figure (4) Correlation between CRP and SLEDAI.

\section{There was a significant correlation}

$$
(\mathrm{r}=0.35 \mathrm{p}=0.01)
$$

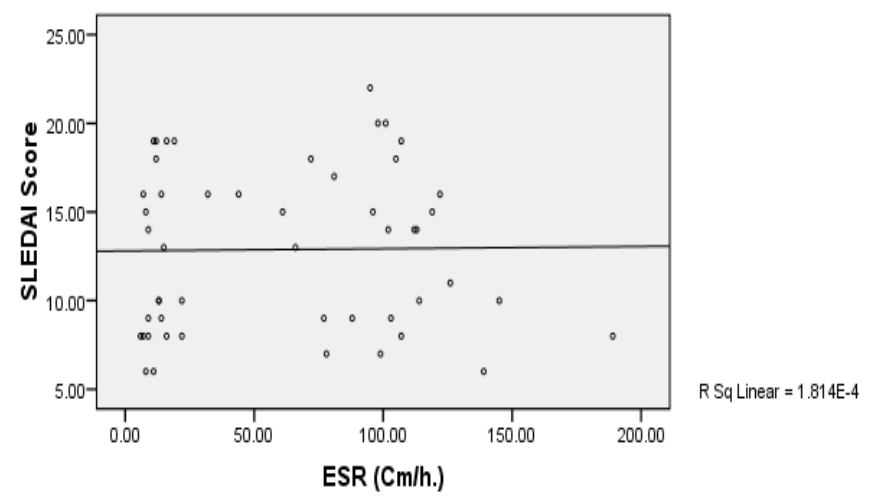

Figure (5) Correlation between ESR and SLEDAI.

There was non-significant correlation

$$
(\mathrm{r}=0.01 \mathrm{p}=0.92)
$$

\section{DISCUSSION}

Patients with SLE have a high risk of cardiovascular disease. Multiple SLE-specific mechanisms, such as autoimmune responses, altered autoantibody, cytokine levels, and lipid dysfunctions can accelerate the progression of overall atherosclerotic burden. Therefore, early detection and quantification of pathological changes are important for assessing the benefits of cardiovascular prevention in SLE management ${ }^{(3)}$. Transthoracic echocardiography can be helpful as a noninvasive diagnostic tool for early detection such abnormalities, resulting in earlier treatment and reduction in mortality and morbidity rates (7). Also the respiratory system is more commonly affected in SLE than in any other systemic autoimmune disease and that all its components may be affected ${ }^{(4)}$. Currently, CT is the best imaging technology in diagnosing the chest lesions in SLE patients. Chest lesions occur with high frequency, and the CT manifestations are complex and various, pulmonary interstitial changes is the most common ${ }^{(8)}$.

In the present study, the most common echocardiography finding was pericardial effusion seen in $40 \%$ of patients, followed by mitral regurgitation in $28 \%$ of patients, mitral valve prolapse (MVP) was seen in 26\% of patients. The other echocardiography findings were aortic regurgitation in $6 \%$ patients, tricuspid regurgitation and pulmonary hypertension in $8 \%$ of patients each. Systolic dysfunction and diastolic dysfunction in $10 \%$ of patients each. Regional hypokinesia was seen in $12 \%$ of patients.

This was in agreement with Purushottam et al. ${ }^{(9)}$ who did a study included 50 patients of systemic lupus erythematosus of whom 44 patients were females and 6 patients were males. Majority of patients were between the age group 20 to 40 years. The maximum prevalence of systemic lupus erythematosus was seen in the age group of 31 to 50 years. Echocardiography was done in all patients and they found the same results as in our study. Furthermore, they found one patient had LibmanSacks endocarditis and MVP with mitral regurgitation seen in 10 patients. 
In another study by Shazzad et al. ${ }^{(10)}$, fifty lupus patients were enrolled in the study and were evaluated by standard echocardiography with color Doppler. SLEDAI was applied for assessment of disease activity. Out of 50 patients $80 \%$ had abnormal echocardiographic findings. Pericardial thickening was found in 19 patients (38\%), pericardial effusion in 10 patients (20\%), diastolic dysfunction in 36 patients $(72 \%)$, hypokinesia of ventricular wall in 4 patients $(8 \%)$, overall valvular abnormalities $20 \%$, commonest being aortic regurgitation in 6 patients (12\%), followed by mitral regurgitation in 4 patients $(8 \%)$, pulmonary hypertension in 3 patients $(6 \%)$. In our study, there was a significant correlation between disease duration and EF\% $(p=0.02)$. This was in agreement also with Shazzad $\boldsymbol{e t}$ al. ${ }^{(\mathbf{1 0})}$, as they found significant relationship between disease duration and cardiac abnormalities $(\mathrm{p}<0.01)$.

As regard CT chest in our study, there was 13 patients (26 \%) had normal CT chest. The most common CT chest findings was ground glass opacity seen in 15 patients $(30 \%)$ followed by pleural effusion seen in 14 patients (28\%), pleural thickening in 10 patients (20\%), interlobular septal thickening in 9 patients (18\%) and mediastinal lymph node enlargement, pulmonary artery trunk broadening, bronchiectasis, consolidation in 5 patients $(10 \%)$ each, honey comb opacity in 4 patients (8\%) and emphysema in one patient (2\%).

This was in agreement with Ping $\boldsymbol{e t}$ al. ${ }^{\left({ }^{(8)}\right.}$ who did a study included 39 patients ( 34 females and 5 males), aged from 19 to 74 years old, the mean age was $(44.13 \pm 12.17)$ years old. HRCT chest was done for all patients and they found the same results as in our study. Furthermore, they found funicular opacity in 20 patients, sub-pleural line in 9 patients and mosaic sign in 3 patients.

In other study by Kakati et al. ${ }^{(11)}, 38$ lupus patients, were enrolled in the study and were evaluated by HRCT chest. The age of the patients ranged from 12 to 60 years (mean age 26.18 years) with maximum incidence between 16 to 25 years of age and 3 patients $(7.89 \%)$ were males. Pleural effusion was seen in 4 patients $(10.53 \%)$, pleural thickening in one patient $(2.63 \%)$, sub-pleural bands in 2 patients $(5.26 \%)$, lymphadenopathy in 2 patients $(5.26 \%$ ), bronchiectasis in 3 patients $(7.89 \%)$, interlobular septal thickening in 15 patients (39.47\%), parenchymal bands in 14 patients (36.84\%), air space consolidation in 2 patients $(5.26 \%)$, ground glass opacification in 10 patients ( $26.32 \%$ ), parenchymal micronodules in 3 patients (7.89\%), and bullae in 2 patients $(5.26 \%)$.

\section{CONCLUSION}

It is common to find cardiac and chest involvement in SLE patients. All SLE patients even who have clinically inactive disease should be screened for the presence of structural cardiac and chest abnormalities. Trans-thorasic echocardiography and CT chest can be helpful as a noninvasive diagnostic tool for early detection of such abnormalities, resulting in earlier treatment and reduction in mortality and morbidity rates.

\section{REFERENCES}

1-James W, Berger TE and Drink A (2005): Diseases of the skin: Clinical Dermatology. 10 ${ }^{\text {th }}$ Ed; Pp. 1100-1107.

2-Rahman A and Isenberg A (2008): Review Article : Systemic Lupus Erythematosus. North England Journal of Medicine, 358 (9): 929- 39.

3-Lin K, Donald M, Lioyd J et al. (2016): Imaging of cardiovascular complications in patients with systemic lupus erythematosus. Lupus, 24(11): 1126-34.

4-Salinas M J, Caeiro F, Saurit V et al. (2017): Pleuropulmonary involvement in patients with systemic lupus erythematosus from a Latin American inception cohort. journals.sagepub.com; Lupus ,50: 1-10.

5-Lapteva L, Nowak $M$ and Yarboro $C$ (2006): Anti-Nmethyl-D-aspartate Receptor antibodies, cognitive dysfunction, and depression in systemic lupus erythematosus. Arthritis Rheum., 54:2505-14.

6-Rahman P, Gladman D, Urowitz $M$ et al. (2001): Early damage as measured by SLICC/ACR damage index is a predictor of mortality in systemic lupus erythematosus. Lupus, 10(2):93-6.

7-Abdel Gaffar A, Abdulaziz A, Mohammad A et al. (2017): Echocardiographic findings in asymptomatic systemic lupus erythematosus patients. Clin Rheumatol., 36:563-8.

8-Ping Li , Shenghong Li , Lan Li et al. (2017): Chest CT findings in systemic lupus erythematosus and its correlation with serum markers. Radiology of Infectious Diseases, 4: $7-$ 13.

9-Purushottam Rao B, Lakshmi $R$ and Satyanarayana V (2015): "Cardiovascular Complications in Systemic Lupus Erythematosus: A Study in A Tertiary Care Hospital". Journal of Evidence based Medicine and Healthcare, 2(35): 5348- 54.

10-Shazzad MN, Islam MN, Ara $R$ et al. (2013): Echocardiographic assessment of cardiac involvement in systemic lupus erythematosus patients. Mymensingh Med J., 22(4):736-41.

11-Kakati S, Doley B, Pal S et al. (2007): Pulmonary Manifestations in Systemic Lupus Erythematosus with Special Reference to HRCT. Japi., 55:839-841. 